\title{
The Effect of TLS Radome on GNSS Precise Positioning
}

\author{
Dhota Pradipta $^{1}$, Dudy D. Wijaya ${ }^{1}$,Heri Andreas ${ }^{1}$, and Dina A. Sarsito ${ }^{1}$ \\ ${ }^{1}$ Geodesy Research Group, Faculty of Earth Sciences and Technology, Institute of Technology, Bandung, Indonesia.
}

\begin{abstract}
This article presents the results of a test carried out to check the usability of spherical TLS targets as GNSS antenna radomes (herein called TLS radomes). On different days, the survey was conducted using two GNSS antennas, one of them with a TLS radome. Measurements were made using 2 roof pillars on the rooftop as base-rover pillars with little obstruction. The measurements were carried out for approximately 1 hour in every scenario. The software used for data processing is MATLAB-based software and the raw data were processed using the double difference (DD) strategy to obtain optimal results. The results of the data processing indicated that the TLS radome has no significant influence on availability and accuracy of estimated position. The signals are slightly attenuated by the radome $(1-2 \mathrm{dBHz})$ and the noise level is slightly increased but both effects are negligible for practical purposes. However, we found that the antenna should be calibrated with the TLS radome to clarify apparent minor phase center eccentricities (1-2 $\mathrm{mm})$ and to reduce systematic effects with long periods (few minutes) and amplitudes up to about $5 \mathrm{~mm}$ which are likely due to phase center variations.
\end{abstract}

\section{Introduction}

Use The use of TLS targets is required for absolute orientation of point clouds obtained by laser scanning. It is particularly important for change monitoring where such targets can be used to establish the connection to a well-defined datum. The experiment presented herein is based on the idea of mounting a GNSS antenna inside a TLS target so that its position can be determined precisely while scanning. The spherical TLS target resembles an antenna radome. Therefore we will call it"TLS radome" within this report. The experiments were carried out at the rooftop for collecting the data. The purpose of the observations is to determine the effects of the TLS radome on the positioning. In particular we want to clarify

1. whether GNSS positioning is possible using the TLS radome (signal reception sufficient);

2. whether the TLS radome increases the noise level of the raw observations and the computed positions (P3 and P5), and lastly,

3. whether there are any systematic effects on the antenna phase center and phase center variation requiring calibration.

Two pillars were selected for use because they have little obstruction (P3, P5). Observations were carried out on 4 days and during periods with approximately equal satellite geometry.

For scenario 1 of the experiment, we used two NovAtel $702 \mathrm{GG}$ antennas, one without any modification and one mounted inside the TLS radome but without the top half of the radome. We put the GNSS antenna with the half open TLS radome on pillar P5 as a rover and the other one as base on pillar P3. Then, for scenario 2, data were collected using a closed TLS radome on P5 and the same antenna as before on P3. For scenario 3, we swapped the position of both devices such that the closed TLS radome was now on the base pillar located with fixed coordinates and the antenna without TLS radome is the rover. And lastly, we measured without any TLS radome and compared the results to those of the other scenarios.

The processing was carried out using in Matlab and the measurement data were taken from download in the rinex form. Satellite orbits were retrieved from the IGS server. Antenna phase center data were retrieved from NGS. In this article it is assumed that the reader has a basic understanding of GNSS and GNSS data processing.

\section{GNSS Measurement}

\subsection{Measurement Equipment}

Measurements were made using the same equipment and sites in the four scenarios with four days of measurement, as outlined below in table 2.1 and figure 2.1 


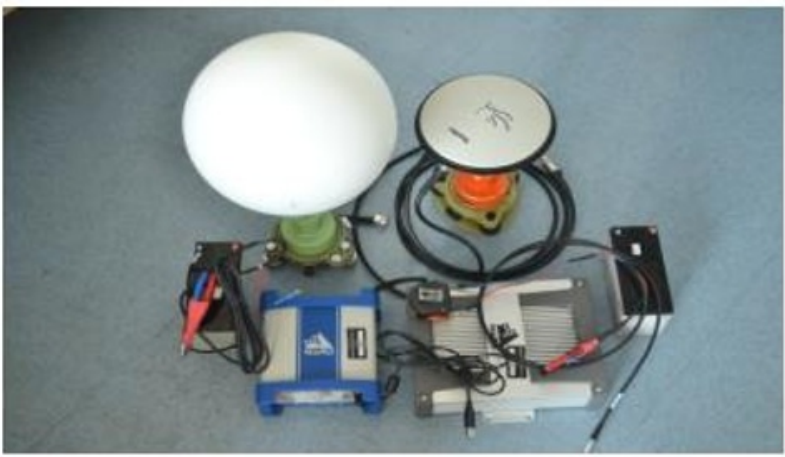

Figure 2.1 GNSS Equipment

Table 2.1. GPS Equipment dan sites used

\begin{tabular}{|l|l|}
\hline Receiver & $\begin{array}{l}\text { 2 Receiver dual frequencies } \\
\text { Novatel DL-V3 and SPAN SE-S }\end{array}$ \\
\hline Antenna & 2 Novatel antennas NOV702GG \\
\hline Power Supply & 2 batteries/Power supply \\
\hline Site & 2 Sites (P3 and P5) \\
\hline Orientation & $\begin{array}{l}\text { Antenna orientation approx. toward } \\
\text { north }\end{array}$ \\
\hline Data rate & 1 second epoch data rate observation \\
\hline
\end{tabular}

\subsection{Measurement scenarios}

(Scenario 1) - open TLS Radome on Pillar 3

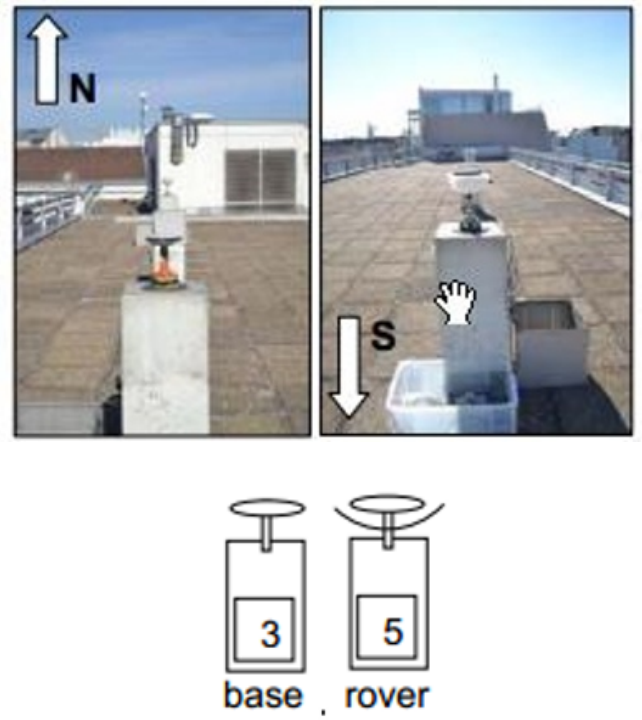

Figure 2.2 North - South view scenario 1
(Scenario 2) - Closed TLS Radome on Pillar 3
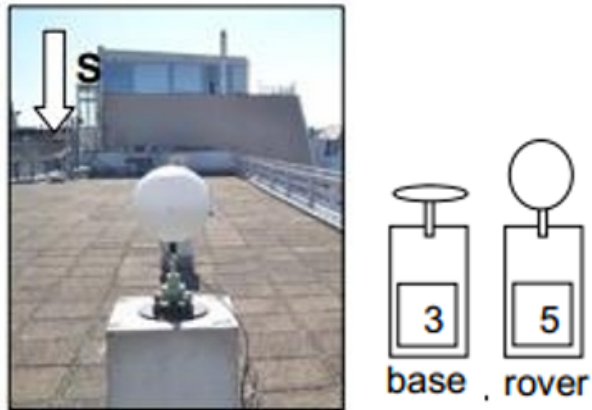

Figure 2.3 South view scenario 2

(Scenario 3) - Closed TLS Radome on Pillar 5
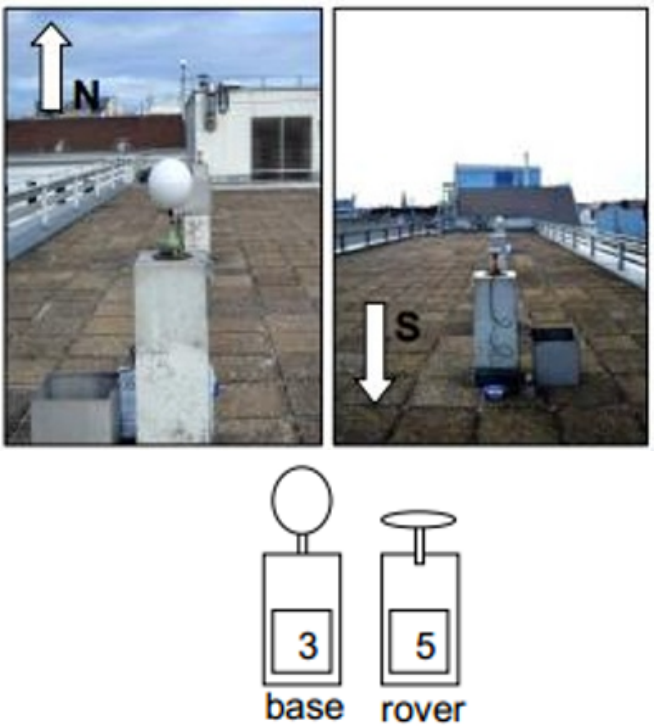

Figure 2.4 North - South view scenario 3

(Scenario 4)-Without TLS radome
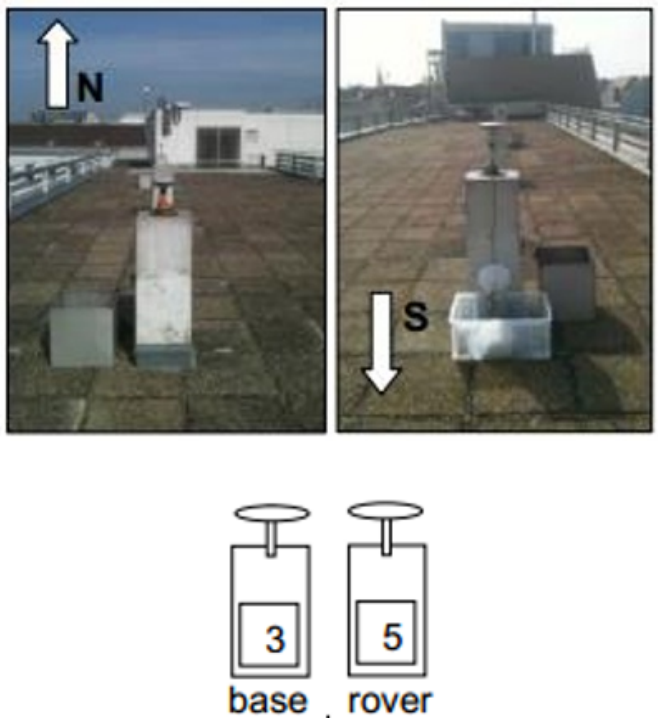

Figure 2.5 North - South view scenario 4 
The environment and obstruction view around the two points of observation are given in figures 2.6 and 2.7

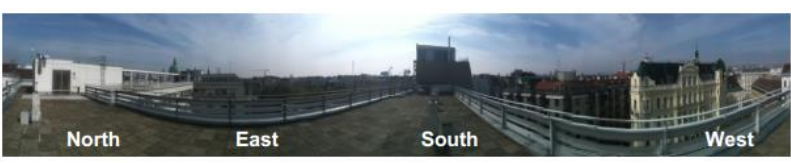

Figure 2.6 Panoramic View of surrounding area sites
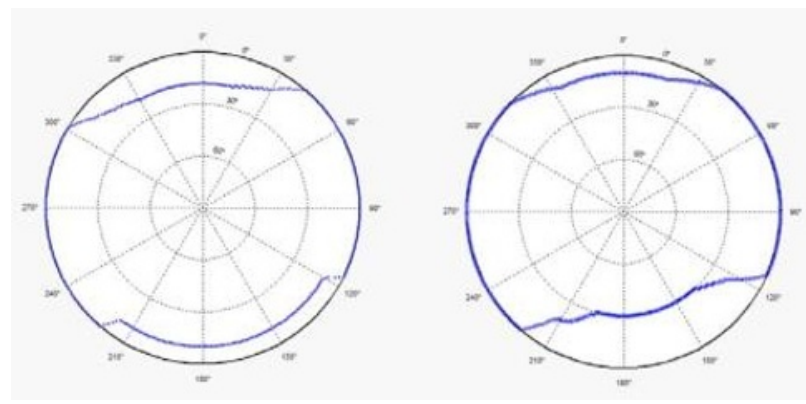

Figure 2.7 Obstruction view on Observation pillar 5 (right) and pillar 3 (left).

The measurement data and the required auxiliary data are as given in table 2.2 .

Table 2.2 Data for data processing

\begin{tabular}{|l|l|}
\hline $\begin{array}{l}\text { Observation Data } \\
\text { (RINEX) } \\
\text { (PLAA = P3 fixed } \\
\text { coordinate) } \\
\text { PLAB = P5 coordinate) }\end{array}$ & $\begin{array}{l}\text { DOY 089, DOY 090, } \\
\text { DOY 093, DOY 094 }\end{array}$ \\
\hline $\begin{array}{l}\text { IGS data } \\
\text { Ultra rapid orbit file }\end{array}$ & $\begin{array}{l}\text { igs16814.sp3, } \\
\text { igs16815.sp3, } \\
\text { igs16821.sp3, } \\
\text { igs16836.sp3. }\end{array}$ \\
\hline $\begin{array}{l}\text { Auxiliary data } \\
\text { (updated using data } \\
\text { from NGS) }\end{array}$ & $\begin{array}{l}\text { receiver information file } \\
\text { satellite problem file } \\
\text { noise model parameter file } \\
\text { information coordinate file }\end{array}$ \\
\hline
\end{tabular}

\subsection{Data Processing and methodology}

This article investigates the relative positioning performance. In general, the observed data from satellites can be defined as follows [1]:

$$
\begin{aligned}
& P_{i}=\rho+d \rho+d_{\text {trop }}+d_{\text {ion }}+c(d t-d T)+M P_{i}+\vartheta P_{1} \\
& L_{i}=\rho+d \rho+d_{\text {trop }}-d_{\text {ion }}+c(d t-d T)+M L_{i}+\lambda_{i} N_{i}+\vartheta L_{i}
\end{aligned}
$$

where $P_{i}$ and $L_{i}$ are pseudorange and carrier phase range on selected frequency $(i=1,2), \rho$ is related to geometrical range from receiver to satellite, $d \rho$ is the orbital error, $d_{\text {trop }}$ and $d_{\text {ion }}$ are troposphere and ionosphere biases, $c$ is the speed of light $(299,729,458 \mathrm{~m} / \mathrm{s}), d t$ and $d T$ are time error of receiver and satellites, $M P_{i}$ and $M L_{i}$ are related to multipath error of pseudorange and carrier phase range, $\lambda_{i}$ and $N_{i}$ are related to wavelength and ambiguity number, while $\vartheta P_{i}$ and $\vartheta L_{i}$ are related to noise error.

\section{Relative Positioning}

The performance of relative positioning were analyzed by using double difference (DD) positioning using carrier phase range that constructed by differencing two single difference (SD) observation. SD can be described as follows:

$$
\begin{array}{r}
\Delta L_{A, B}^{j}=\Delta \rho_{A, B}^{j}+d^{j}{ }_{\text {tropA }, B}-d^{j}{ }_{i o n A, B}+c(d t-d T)^{j}{ }_{A, B} \\
+M L_{A, B}^{j}+\lambda N_{A, B}^{j}+\vartheta L_{A, B}^{j}
\end{array}
$$

where $\Delta$ is the difference between receivers $\mathrm{A}$ and $\mathrm{B}$. The superscript $-j$ is the observed satellite. The satellite clock error is eliminated by taking single difference between receivers that observed the same satellite, while the atmospheric biases like tropospheric and ionospheric may be eliminated depending to the length of the baseline. Multipath would be considered as a noise error that could not be eliminated.

The remaining receiver clock error is then eliminated by subtracting two single difference observation. Mathematically, a DD is defined as follows [2] [6]:

$$
\nabla \Delta L_{A, B}^{j k}=\Delta \rho^{j k}{ }_{A, B}++M L^{j k}{ }_{A, B}+\lambda N^{j k}{ }_{A, B}+\vartheta L^{j k}{ }_{A, B}
$$

where the two satellites are denoted as he superscript $-j$ and $k$. The atmospheric biases are negligible in Eq. 3, however the noise error is multiplied up to two times with respect to SD. The processing strategy was designed as follow:

- The following calculations were carried out using data from observation, i.e. 1 hour of observation Rinex data 1 second sampling rate. Calculations were also carried out with Glonass signals (if available) during similar observation periods in equal satellite geometry. Integer ambiguities are estimated and resolved on an epoch-by-epoch basis in kinematic positioning mode.

- The initial scenario assumes all the observations are processed in difference mode. Hence, the atmospheric errors and clock errors are almost canceling. To create a scenario with different radome, the data were sorted out by the stations to arrange the conditions required. As the local time and the location of the test are essentially affecting the results due to variations in the ionosphere and troposphere activity, calculations of the measurement effect on same time window sessions were performed.

- Mask angles of ten degrees were selected. The receiver noise level was neglected, and when required, IGS precise orbit corrections were used. Tropospheric effects are also independent 
of frequency. Hence a Saastamoinen model was used as the standard model correction [3].

- Furthermore, the observations were grouped in short-term observations (less than and equal to 60 minutes) for positioning in particular to study with the impact of temporal variability for static and kinematic positioning.

- Additionally, since most observation data covers satellites with low elevation angles, observation to satellites which are below 10 degrees are removed to minimize the influence of errors in the data processing.

- The distance between base and rover was under 50 meters. By using this short baseline, the atmospheric errors are assumed to be eliminated.

- The LAMBDA ambiguity resolution search was used to estimate the carrier phase ambiguities [8]. The final solution was the combined (Forward + Backward filter).

\section{Results}
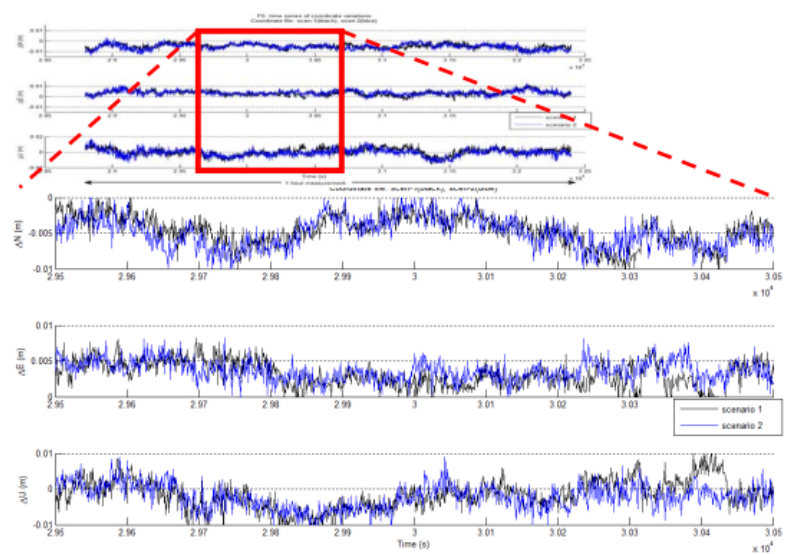

Figure 3.1 Comparison of the position between scenario 1 and 2 on pillar 5.

The results of the data processing show that both highand low-frequency noise affects the estimated position. To further analyse, we compare the time series obtained for times with corresponding satellite distribution (figure 3.1 and 3.2).

Figure 3.1 shows the North-, East- and Up-coordinate variations as obtained from kinematic processing of the measurements from scenario 1 (black) and 2 (blue). The TLS radome was closed in scenario 2 but half open in scenario 1 . The figure shows that there is slightly more high-frequency noise with closed TLS radome than using the half-open one. However, when computing the standard deviation of the kinematic coordinates, the lowfrequency noise which is the effect of multipath interference and phase center variations dominates [5],[7],[9]. The level of these low-frequency components is not changed when the TLS radome is closed, see table
3.1. We conclude that there is no significant effect of the top half of the TLS target on the quality of the estimated position in case the position is determined from static processing of several minutes of data.

On the other hand, the time series exhibit equal noise level of both high- and low- frequency contributions before and after swapping the antennas i.e., for scenarios 2 and 3. This is expected. However, we also note that there is a mirrored systematic error pattern (figure 3.2). This is an indication of systematic errors due to unmodelled phase center variations. Since phase center variations of the employed Novatel GPS antennas were used we interpret this as an effect of the TLS radome on the phase center variations.

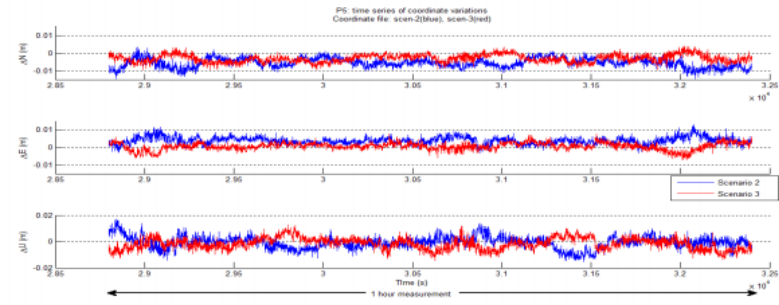

Figure 3.2. Comparison of the position between scenario 2 and 3 on pillar 5 .

From figure 3.1, we know that there is no significant difference in noise level as well as systematic errors with half-open versus closed radome TLS, and also the median values differ by less than $1.0 \mathrm{~mm}(\mathrm{~N}, \mathrm{E})$ and $1.4 \mathrm{~mm}(\mathrm{U})$ (see table 3.2). This indicates that there this is a negligible effect of the top half of the TLS radome on noise and mean phase center.

From figure 3.2, we discovered that scenario 2 and 3 yield equal noise levels and but opposite sign of the systematic pattern. Any mean phase center difference $\Delta \mathrm{p}$ between antenna $\mathrm{w} /$ and $\mathrm{w} / \mathrm{o}$ TLS radome would cause a difference of magnitude $2|\Delta \mathrm{p}|$ of the estimated coordinates before and after antenna swap. In this experiment (see table 3.2) the median values only differ by $1.6 \mathrm{~mm}(\mathrm{~N}), 3.5 \mathrm{~mm}(\mathrm{E})$, and $1.1 \mathrm{~mm}(\mathrm{U})$. So, the phase center eccentricity is most likely affected by less than $2 \mathrm{~mm}$ by the TLS radome.
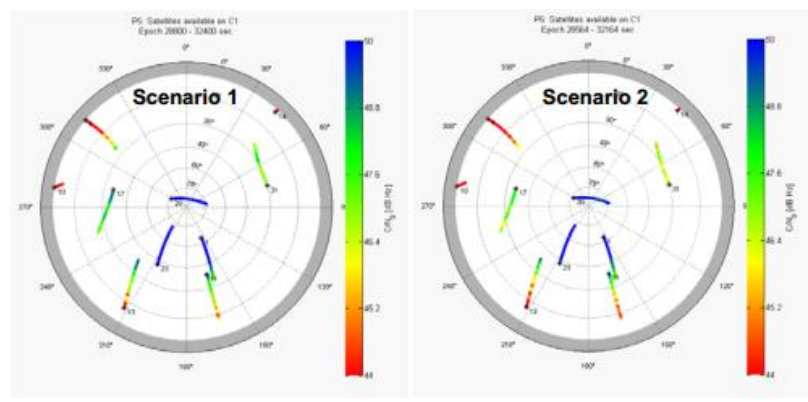

figure 3.3 The sky plot and signal strength of observation on pillar 5 (scenario 1 and 2) 

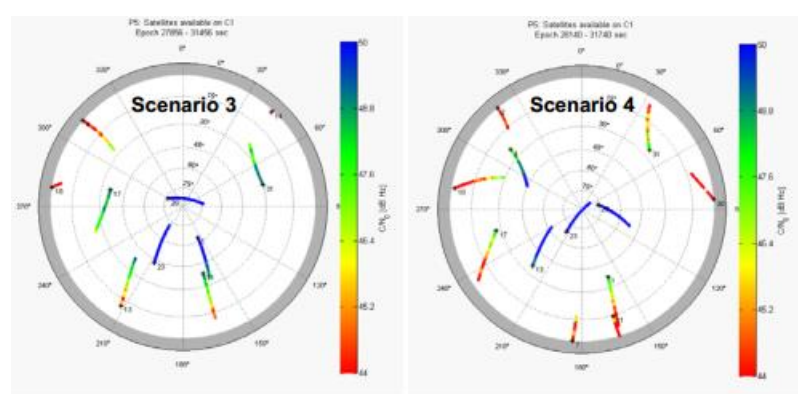

figure 3.4 The sky plot and signal strength of observation on pillar 5 (scenario 3 and 4)

Figure 3.3 and 3.4 indicate a change in signal strength of approximately $1-2 \mathrm{dBHz}$ which is the effect of the TLS radome during the observation. This shows that there is a slight attenuation i.e., an effect on the signal strength, but the effect is small e.g. compared to the effect of satellite elevation on signal strength. So, the TLS radome does not attenuate signals strongly and thus not cause significant problems for GPS signal acquisition and tracking.

Figure 3.5 shows the residuals of individual raw observations. Again, the pattern corresponding to the different scenarios seems to be mirrored with an antenna swap (different colors for different scenarios, as before)
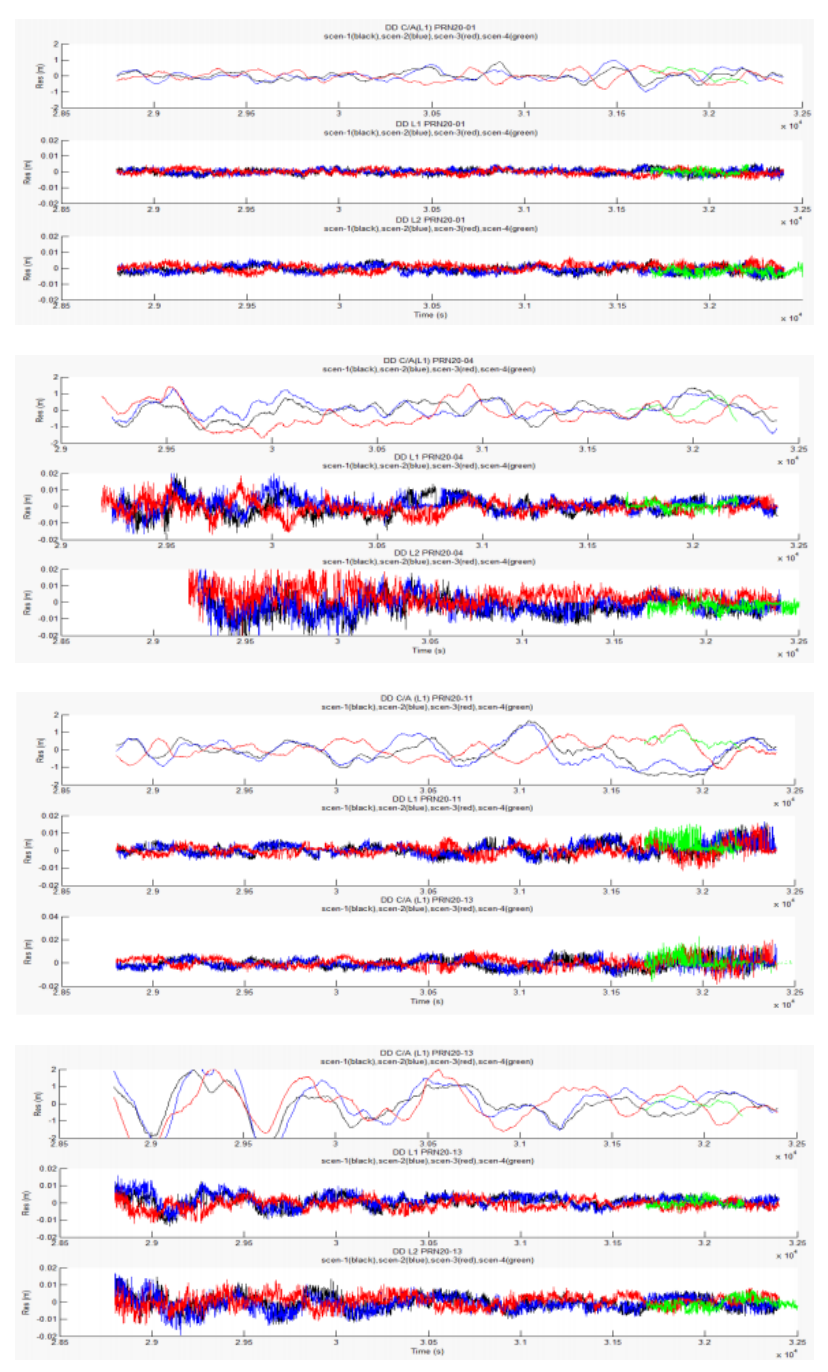

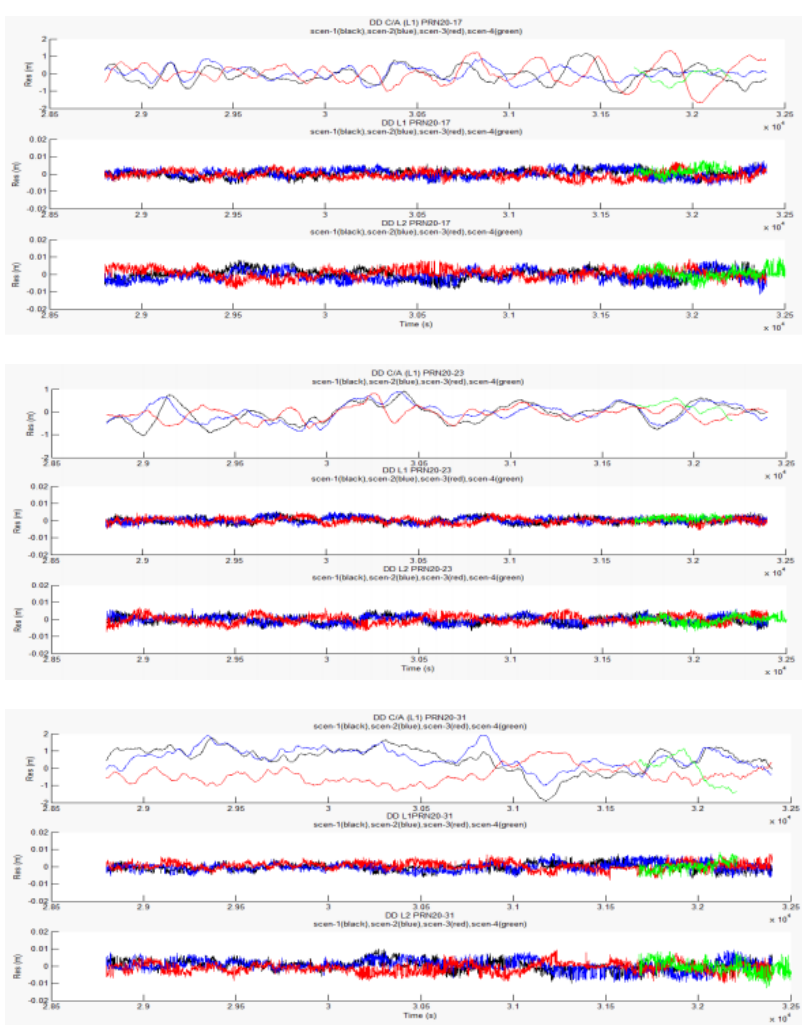

Figure 3.6 PRN residual on satellite 1, 4, 11, 13, 17, 23, and 31 .

Key statistics of the estimated positions are given in tables 3.1 and 3.2.

Table 3.1 Statistic of kinematic coordinates of antenna on P5

\begin{tabular}{|c|c|c|c|c|c|c|}
\hline \multirow{2}{*}{ Scenario } & \multicolumn{1}{|c|}{ Mean difference w.r.t. given coordinates $(\mathrm{mm})$} & \multicolumn{3}{|c|}{ Empirical standard deviation $(\mathrm{mm})$} \\
\cline { 2 - 7 } & $\Delta \mathrm{N}$ & $\Delta \mathrm{E}$ & $\mathbf{\Delta U}$ & $\mathrm{N}$ & $\mathrm{E}$ & $\mathrm{U}$ \\
\hline Scenario 1 & 3.1 & 4.4 & -2.9 & 1.6 & 1.1 & 1.5 \\
\hline Scenario 2 & 2.4 & 4.7 & -4.2 & 1.6 & 1.0 & 1.5 \\
\hline Scenario 3 & 1.0 & 0.9 & -3.3 & 1.6 & 1.0 & 1.5 \\
\hline Scenario 4 & 3.8 & 3.4 & -1.8 & 1.5 & 0.9 & 1.7 \\
\hline
\end{tabular}

Table 3.2 Median coordinates of antenna on P5

\begin{tabular}{|l|c|c|c|c|c|c|}
\hline \multirow{2}{*}{ Scenario } & \multicolumn{3}{|c|}{ Median (m) } & \multicolumn{3}{c|}{$\begin{array}{c}\text { Difference of respective median } \\
\text { w.r.t. scenario 2 (mm) }\end{array}$} \\
\cline { 2 - 7 } & $\mathbf{N}$ & $\mathbf{E}$ & $\mathbf{U}$ & $\mathrm{N}$ & $\mathbf{E}$ & $\mathbf{U}$ \\
\hline Scenario 1 & 4087003.1653 & 1200478.9254 & 4731608.9712 & 0.8 & -0.2 & 1.4 \\
\hline Scenario 2 & 4087003.1645 & 1200478.9256 & 4731608.9698 & 0.0 & 0.0 & 0.0 \\
\hline Scenario 3 & 4087003.1629 & 1200478.9221 & 4731608.9709 & -1.6 & -3.5 & 1.1 \\
\hline Scenario 4 & 4087003.1659 & 1200478.9244 & 4731608.9723 & 1.4 & -1.2 & 2.5 \\
\hline
\end{tabular}

It can be seen from table 3.1, table 3.2 and figure 3.1, figure 3.2 that the precision is approximately equal for all different scenarios. In particular, is the standard deviation is $1.6 \mathrm{~mm}(\mathrm{~N}), 1.0 \mathrm{~mm}(\mathrm{E})$, and $1.5 \mathrm{~mm}(\mathrm{Up})$. However, the coordinates differ by up to $3.5 \mathrm{~mm}$ between the scenarios. Based on the respective empirical standard deviations, these differences are not significant. The duration of the test measurements is too short in order to clarify whether these differences are actually random deviations or are due to phase center eccentricities and systematic effects caused by the TLS radome. Even if they are systematic and due to the TLS radome the effects are small (less than $2 \mathrm{~mm}$ in mean phase center) and may be negligible for the intended application (if the effects are equal for all TLS radomes used within the network). However, it would be appropriate to carry out a proper 
antenna calibration using a TLS radome in order to further clarify this point.

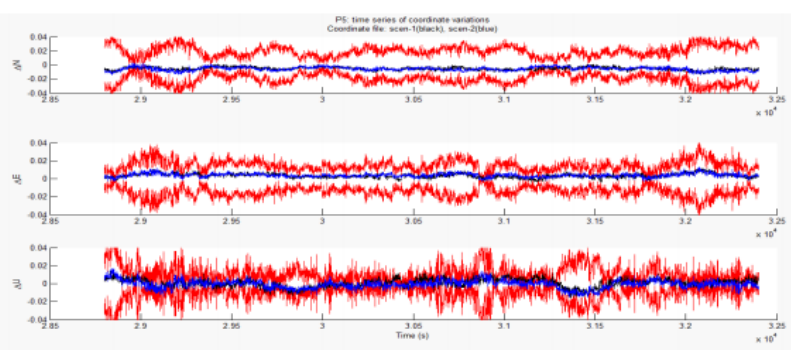

Figure 3.7 Variation of estimated coordinates of site P5

On figure 3.7, we assume that the measurements are normally distributed, that their errors are 0 on average, and that their variance is correctly given by the variance model used for post-processing the raw GPS observations. It means that most of the estimated coordinates $(99.7 \%)$ should be within $\pm 3 \sigma$ of the true coordinates. Using the given coordinates as a reference and plotting the deviations of the estimated coordinates from the true ones (blue) and the respective $\pm 3 \sigma$ bounds about the true coordinates (red), we see in the figure that most of the time the estimated coordinates are within the error bounds and thus there is no need to further investigate the errors. It shows on figure 3.8 that on every scenario, the residuals of double difference are less than $2 \mathrm{~cm}$.
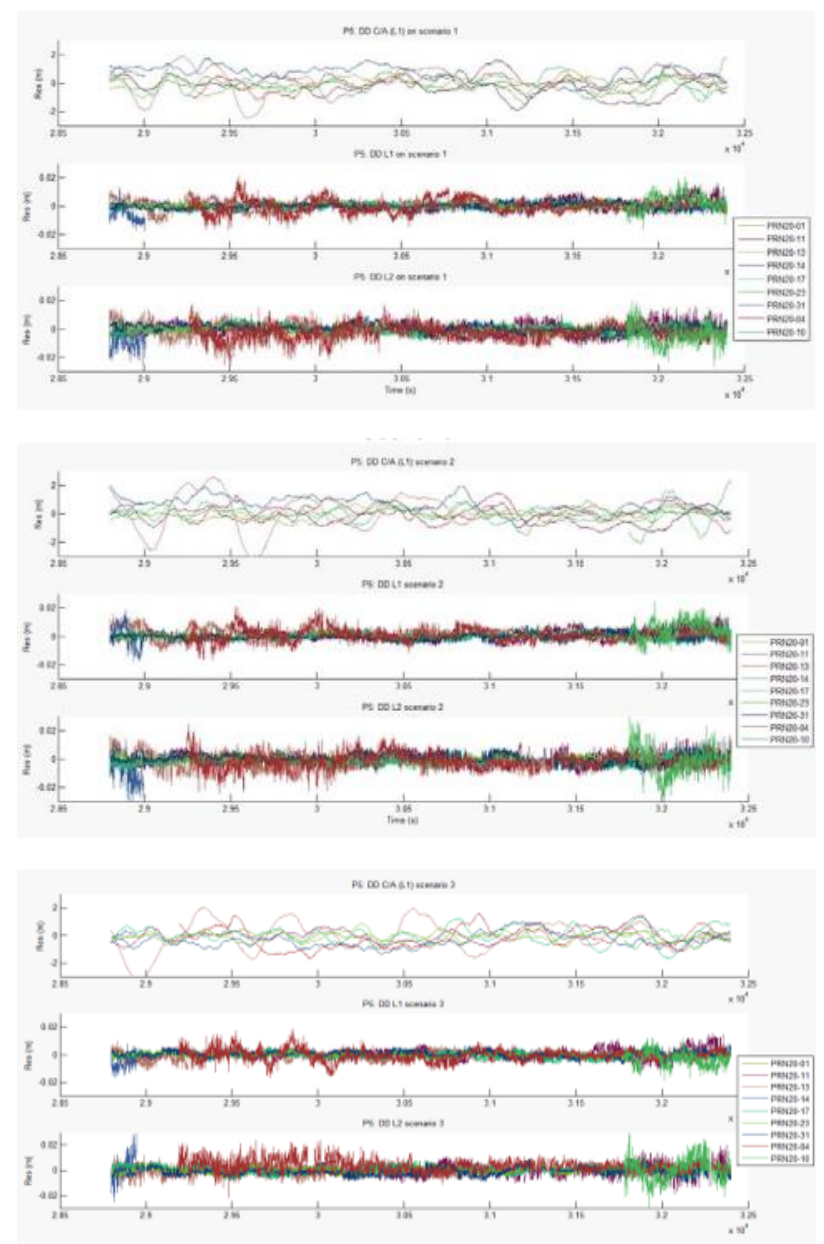

Figure 3.8 Time series of Double Difference Residuals from scenario 1,2 , and 3

\section{Conclusion}

The use of the TLS radome for GPS measurements slightly attenuates the signals (approximately $1-2 \mathrm{dBHz}$ ). However, this effect is small and does not impair the availability of GPS position solutions. GPS positioning is possible using this TLS radome and standard GPS data processing.

The precision obtained for kinematic positions in the different scenarios is about $1.6 \mathrm{~mm}(\mathrm{~N}), 1.0 \mathrm{~mm}(\mathrm{E}), 1.5$ $\mathrm{mm}(\mathrm{U})$ for all scenarios. Using the TLS radome does not significantly increase the noise level of the coordinates when processing data in static or short-term static mode (at least a few minutes of data).

We found mirrored systematic error patterns when swapping the antennas with and without TLS radome. The error patterns have a magnitude of up to $5 \mathrm{~mm}$. The median coordinates over 1 hour changed by less than 4 $\mathrm{mm}$ between the scenarios. All this indicates that there may be a systematic effect of the TLS radome on mean phase center and on phase center variation on the mmlevel. Given this order of magnitude, these effects would be negligible for the proposed application if the same TLS radome/GPS antenna were always used on the same network sites, the GPS antennas and radomes were always oriented equally, and GPS positions were derived from static or short-term static sessions only.

However, this may not be feasible from a practical point of view, and it is not possible to predict whether or not different TLS radomes would cause the same phase center eccentricities and variations as the radome used herein. Thus, we propose to calibrate 2-3 GPS antennas with TLS radome in oder to find out, whether these effects are actually systematic and predictable across different samples or not. The results of such a calibration (in particular: repeatability of parameters for the same antenna/radome, repeatability across antennas/radomes, order of magnitude of parameters) will allow selecting an appropriate site setup and data processing scheme.

Should the design of the TLS radome be changed, e.g. by selecting a different TLS target shape/size or material, or by coating the target for modified optical reflectivity, a test resembling scenario 2 and 3 of the experiments covered herein (i.e., static processing with antenna swap) should be carried out to quickly assess the impact. The corresponding data processing can be carried out using standard GPS post-processing software like Leica GeoOffice, Trimble Business Center, or others.

\section{References}

[1] Xu, G. (2007). GPS Theory, Algorithms and Application. Berlin: Springer.

[2] Kaplan, E. D. (1996). Understanding GPS: Principles and Applications. London: Artech House Boston.

[3] Amiri-Sikooci, A., Tiberius, C., C., J., M. (2007). Assessing Receiver Noise Using GPS Short Baseline Time Series. GPS Solution, Vol. 11. Pp21-35 DOI 10.1007/s10291-006-0026-8.

[4] Davaine, M. (2011). Code Bias and Multipath Estimation with Cascaded Kalman Filter. Thesis. Insitute for Communication and 
Navigation, Technische Universitaet Muenchen. Munich, November.

[5] Dawidowicz, K. (2010). Antenna Phase center Variations Corrections in Processing of GPS Observations with the use of commercial Software. Technical Science. No 13, DOI 10.2478/v10022-010-0012-9.

[6] Hofmann-Wellenhof, B., Lichtenegger, E. Wasle. (2008). GNSSGlobal Navigation Satellite Systems. Springer, Wien.

[7] Joseph, A. and Petovello, M. (2010). Measuring GNSS Signal Strength. Inside GNSS. November, pp. 20-25.

[8] Teunissen, P. J. G., (1995). The Least-square Ambiguity Decorrelation Adjustment: A Method for Fast GPS Ambiguity Estimation. Journal Geodesy, vol.70.

[9] Wu, J.T., S.C. Wu, G.A. Hajj, W.I. Bertiger, and S.M. Lichten. (1993). Effects of antenna orientation on GPS carrier phase. Manuscripta Geodaetica. Vol. 18. pp. 91-98. 